\title{
Option of the Optical-mechanical Positioning of the Sensor and Sample in the Magnetometer. Determination of Magnetic Susceptibility of Powders and particles
}

\author{
Alexander Sandulyak ${ }^{1}$, Anna Sandulyak ${ }^{1,{ }^{*}}$, Dmitriy Kiselev ${ }^{1}$, Maria Polismakova ${ }^{1}$, and Hasan \\ Bulut $^{2}$ \\ ${ }^{1}$ Moscow technological university, Moscow, RF \\ ${ }^{2}$ Department of Mathematics, University of Fırat, Elazı ğ, Turkey
}

\begin{abstract}
In Faraday's magnetometer it is recommended to apply remotely the located poles hemispheres. In this case (and similar cases) there is a need for expeditious and exact positioning of the measuring sensor (Hall) and the studied sample - for obtaining the most authentic characteristics of induction and its gradient, identification and use of a zone of stability. This problem is solved by the corresponding opticalmechanical system of positioning. It consists of the laser modules promoting aim positioning, the Web camera promoting final positioning. On the received concentration dependences of magnetic susceptibility of powder samples existence of limited line sections is confirmed that gives the chance to define susceptibility of particles. The critical relation to use for the similar purposes of samples (disperse) in the form of colloids and suspensions expresses.
\end{abstract}

\section{Introduction}

Faraday's ponderomotive method is preferable in a case of statement of the problem about determining a sample' s magnetic susceptibility $\chi$ with a small volume $V$. Herewith it could be both solid and disperse sample (for example, powder sample of ferro- or ferromagnetic particles, which are disperse phase of many technological mediums, including those which are affected by magnetophoresis and magnetic control [1-6]). A magnetic (ponderomotive) force $F$, acting on study "microsample", becomes basic measurement value when magnetic loop of Faraday balance of field with intensity $H$ (induction $\left.B=\mu_{0} H\right)$ and nonuniformity $\operatorname{gradH}(\operatorname{gradB})$ is creating between pole pieces. Considering that its functional view, as a rule, is determining by expression:

$$
F=\mu_{0} \cdot \chi \cdot V \cdot H \operatorname{grad} H=\chi \cdot V \cdot \operatorname{BgradB} / \mu_{0},
$$

\footnotetext{
* Corresponding author: anna.sandulyak@mail.ru
} 
where $\mu_{0}=4 \cdot 10^{-7} \mathrm{H} / \mathrm{m}$ - magnetic constant, so having known (measured) values of expression' s parameters and using subsequent calculating formula:

$$
\chi=\frac{F}{\mu_{0} V \operatorname{HgradH}}=\frac{F \mu_{0}}{V \operatorname{VgradB}}
$$

quantitative information about $\chi$ becomes available.

Placement of the sample in a certain (working) zone of the interpolar region, in which the field nonuniformity must be constant [7-13], is considered an important condition for the implementation of the method. Proper attention has not been paid to this problem at the literature. Existing recommendations about choosing a configuration of pole pieces are greatly general, still within question about the necessity for distinct sample positioning.

\section{Discussion and results}

The coordinate characteristic of parameter $H$ or $B$ is needed for objective estimation about acceptance of choosing pole pieces and for having an information about, for example, parameter's behavior of the grad $B(\operatorname{grad} H)$ in one $x$-direction or another at interpolar region (for example, in direction of action of the ponderomotive force). Hall sensor, in particular, is completely applicable for obtaining such characteristic, moreover essentially in detail, with small spacing (in choosing $x$-direction). It is this characteristic $B$, presented at graphic or/ and analytical form, being basic, lets getting coordinate characteristic, for example, parameter grad B. Then it can be inferred, at first, by presence (or absence) of a region of interest (i.e. region with constant $\operatorname{grad} B$ values) in a certain interpolar region and, in second, by coordinates of its location and thus coordinates of sample positioning.

So, if the case in hand is diagnostics of interpolar region using parameter $B$ in one $x$ direction or another (for example, direction of force $F$ action) for finding a region with a stable value of $\operatorname{gradB}$, it is enough to identify a linear (close to linear) segment, even short (comparable with sample' $\mathrm{s}$ dimensions) on the obtained dependence of $B$ on $x$. The presence of such a segment means that $\operatorname{grad} B=d B / d x=$ Const at this location. In this regard it must be admitted that obtaining and analysis of dependence $B$ on $x$ ( $H$ on $x$ ) are outstanding necessary step for realization of the method.

Herewith it should not go unspoken that these dependences are nonlinear in whole for pole pieces with traditional and specific form. This fact disagrees an approach in finding linear segment of dependence of $B$ (or $H$ ) on $x$ in some way.

The essence of a simple and affordable approach for excluding this disagreement consists in selecting pole pieces shaped such that the key characteristic $B(x)$ or $H(x)$ is tortuous between them, i.e., has an inflection. Then, its segment (which is short relative to the entire unfolded nonlinear characteristic) in the vicinity of the inflection point can be considered close to the desired linear one - with almost stable value of $\operatorname{gradB}(\operatorname{gradH})$. In this case the corresponding derivative of the characteristic $B$ (or $H$ ), i.e., the characteristic $\operatorname{gradB}(\operatorname{gradH})$, has an extremum here; it is the neighborhood of this extremum that is an indication of the presence of a zone with an invariable gradB $(\mathrm{gradH})$ value.

Results of the theoretical and experimental researches about magnetizing (by field with intensity $H_{0}$ ) chain of balls point to acceptable (and easy-designed) form of pole pieces [14-17] (Fig. 1a). Precisely these nonlinear characteristics of intensity $H\left(H>>H_{0}\right)$, having an inflection, are representative of region between opposite spherically shaped surfaces in the direction $x$ (Fig. 1a) - from line between centers of balls and normal to it (along "bisecting line" of wedgegap between balls). So, there is a distinct extremum of characteristic $d H / d x$ at the inflection point (Fig. 1b) [14-17]. Consequently, from the standpoint of the creation of a guaranteed working region, the using of spherically shaped pole pieces (Fig. 2) appears to be one of the most effective solutions to the problem of selecting the pole pieces for a Faraday balance [18-20]. 

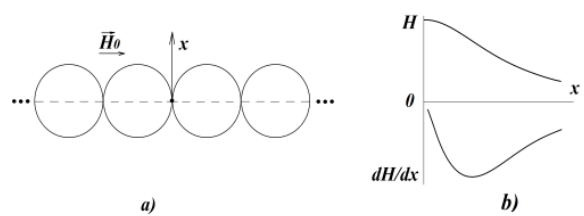

Fig.1. The chain of balls in field of solenoid with intensity $H_{0}(a)$ and corresponding illustration of coordinate (along $x$ ) characteristics [14-17] of intensity $H$ and nonuniformity $d H / d x$ of field at wedgegap between the spherical pole surfaces $(b)$

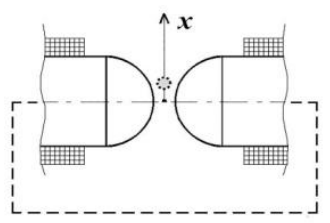

Fig.2. The variant of creation a working region (is located near the abscissa of the inflection of the coordinate characteristic of the magnetic field strength or induction and the abscissa of the extremum of the gradient, correspondingly) between the spherical pole pieces

Figure 3 (points) presents the measured values of induction $B$ in the plane of symmetry of the region between the pole pieces-hemispheres $100 \mathrm{~mm}$ in diameter (separated by 10 $\mathrm{mm}$ ) at different distances $x$ from the axis and different values $I$ of the winding current. The inflection of each characteristic $B(x)$ is clearly seen. Identification of the inflection allows linear approximation of data in the vicinity of the inflection point (shown with dashed lines) that indicates the presence and location of the working region. An extremum view of characteristic grad $B$ indicates the presence, too (Fig. 4).

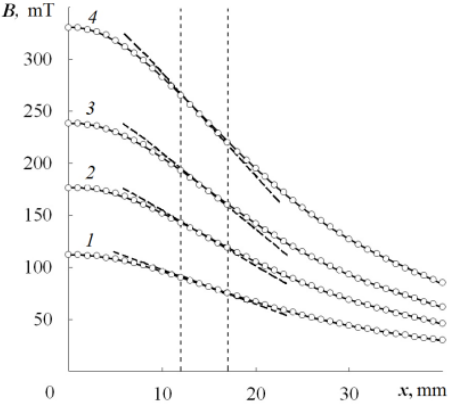

Fig.3. The characteristics of the field induction at the symmetry plane of region between the pole pieces shaped as hemispheres $100 \mathrm{~mm}$ in diameter with the distance between them is $10 \mathrm{~mm}$ for different values of the winding current $I: 1-I=4 \mathrm{~A}, 2$ $-I=8 \mathrm{~A}, 3-I=16 \mathrm{~A}, 4-I=40 \mathrm{~A}$; the dots are the experimental results, the lines present the results of calculation by (3)

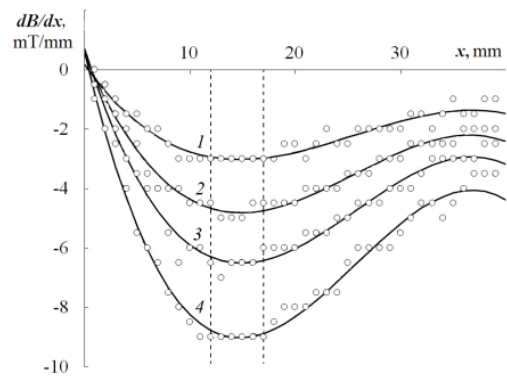

Fig.4. The characteristics of the induction gradient (by data of Fig. $3)$; dashed and solid lines - are the calculation by using equation (4), which is written for polynomial (3)

of 5 th and 4 th degree correspondingly

It should be used analytical (phenomenological) approximation at analysis of characteristic $B$ - with subsequent mathematical differentiation and obtaining gradBcharacteristic herewith obvious identifies an extremum's absciss. In this case it is appropriate using, for example, an approximation function of polynomial [18-20]:

with fourth, maximum fifth degree - it is enough.

$$
B=a_{1} x+a_{2} x^{2}+a_{3} x^{3}+a_{4} x^{4}+\ldots+a_{0}
$$

Analytical dependences $B$ on $x$ such as (3) could be easy differentiate, i.e. $\operatorname{grad} B=d B / d x$ characteristics could be obtained: 


$$
d B / d x=a_{1}+2 a_{2} x+3 a_{3} x^{2}+4 a_{4} x^{3}+\ldots
$$

Dependences (4), which are obtained for polynomial (3) of $5^{\text {th }}$ and $4^{\text {th }}$ degree, are obvious extremal (Fig. 4; dashed and solid lines corresponding), herewith they are in good agreement with each other. The mutual noticeable discrepancy of polynomial (3) of $5^{\text {th }}$ and $4^{\text {th }}$ degree is observed only at high and low $x$ values (Fig. 4). However, taking the fact into account that these values $B$ are not important here a simpler polynomial of the $4^{\text {th }}$ degree is preferable for approximating the $B$ data, and quadrinomial dependence correspondingly (4).

Moreover, it provides an analytical formula for calculating the abscissa of the extremum $x=x_{\text {extr }}$ of the gradB characteristic (Fig. 4) if we based on polynomial (3) of $4^{\text {th }}$ degree. In fact, we could obtain a calculating formula, if we differentiate quadrinomial dependence (4) with subsequent nulling obtained equation of the 2 nd degree, i.e. $a_{2}+3 a_{3} x_{\text {extr }}+6 a_{4}\left(x_{\text {extr }}\right)^{2}=0$ :

$$
x_{\text {extr }}=\left(-3 a_{3}+\sqrt{9 a_{3}^{2}-24 a_{2} a_{4}}\right) /\left(12 \cdot a_{4}\right) .
$$

Formula (5) allows calculating abscissas values of the extrema $x_{\text {extr }}$ of characteristics gradB.

When the current load varies from experiment to experiment the sample location may remain unchanged in the course of the measurements because of abscissas of the extrema are mutually identical (Fig. 3, 4). In this case, the size of the working region in the interpolar area under investigation (between choosing pole pieces), i.e., the region of practically stable $\operatorname{gradB}$ values, is (in terms of $x$ ) $12-17 \mathrm{~mm}$. This is clearly seen in Fig. 3, where the dashed straight lines, as noted above, show the possibility of linearly approximating short segments of dependences $B(x)$ in the vicinity of their inflection point (the corresponding interval of $x$ is marked). This can be seen even more clearly in Fig. 4 on the $x$ interval (it is also highlighted), where the $d B / d x$ value near the extrema of dependences $d B / d x$ on $x$ is relatively stable.

It should be noted that when using pole-hemispherical pieces, additional complications (and with them errors) can arise in the implementation of precise positioning (in particular, in ensuring an accurate starting position), first of all, Hall-effect device. This can adversely affect the accuracy of obtaining the necessary coordinate characteristics of the field, and therefore - the accuracy of determining the working zone of a stable gradient and, as a consequence, the reliability of the result of the definition of susceptibility. We note that even with accurate determination of the working region, it is equally important to realize the exact positioning of the sample.

In this case, it is advisable to use the optomechanical positioning system of the measuring sensor and the sample in the interpolar region. It should include a bi-directional laser module, in particular with a crossed beam for initial (targeted) positioning of the sensor, and a Web camera (connected to a computer), with mutually perpendicular scales for initial and control positioning of the sensor and the sample. Herewith, initial the positioning of the Hall-effect device and its moving in the interpolar region is easy to provide with a three-coordinate table, rigidly connected with the Hall-effect device.

In order to derive the magnetic susceptibility $\chi$ of disperse phase' ferroparticles, with a certain degree of conditionality it is possible to use the famous relationship $\chi=\langle\chi\rangle / \gamma \quad(\langle\chi\rangle$ - magnetic susceptibility of disperse medium).

However, this expression is valid only for rather small values of volume fraction of ferroparticles $\gamma$ (in the volume of studied dispersive medium) - for such volumes, where the mutual magnetic effect of the ferroparticles can be practically excluded. This is possible when the ferroparticles in a sample of the dispersive medium (powder is more preferable instead suspension or colloid in which ferroparticles are susceptible being formed in chains and aggregates [21]) are sufficiently mutually separated. In powder dispersive media, yet another dispersive phase from non-ferromagnetic particles (often obtained after pulverizing sand) is used to achieve a particular $\gamma$ - values (a particular certain degree of dissociation of ferroparticles), which mixed with dispersive phase of ferroparticles. 
Fig. 5 shows the family of dependences of $\langle\chi\rangle$ on $\gamma$ (for various $H$ ) [22]. The dependences are well nigh linear throughout the studied range $\gamma-$ up to $\gamma=0.2-0.25$. If one is guided by the results of linear approximation in this range of $\gamma$, then by relationship $\chi=\langle\chi\rangle / \gamma$ it is possible to determine the values of the susceptibility of particles $\chi$ as a coefficient of proportionality of each dependence from the family of $\langle\chi\rangle$ on $\gamma$ dependences.

The values of $\chi$ as a function of $H$, obtained for the entire set of data, are presented on Fig. 6 in logarithmic coordinates. In such coordinates they are well-quasilinearized, thereby being represented in the specified range of $H$ by a dependence similar to the power function $\left(\chi \sim 1 / H^{0.75}\right)$. With accuracy up to coefficient $K=11400$, it takes the form $\chi=K / H_{x}^{0,75}$ , where $H_{x}=H / 1 \mathrm{~A} / \mathrm{m}$ is the field intensity converted to dimensionless form.

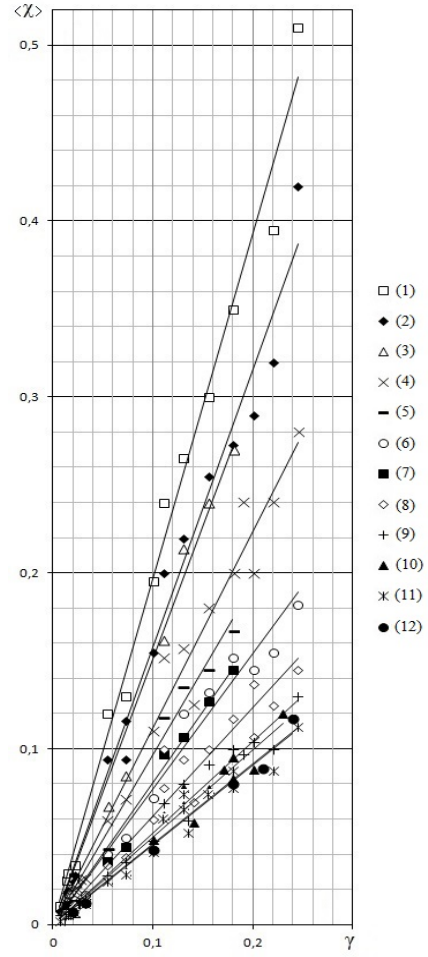

Fig.5. Effect of the volume fraction of magnetite particles in the sample of dispersive medium on its magnetic susceptibility for various values of field intensity $H$ [22]; $1-H=90 \mathrm{kA} / \mathrm{m}, 2-150,3-$ 190, 4- 220, 5- 270, 6- 340, 7- 365, 8- 420, 9- $550,10-650,11-730,12-780$

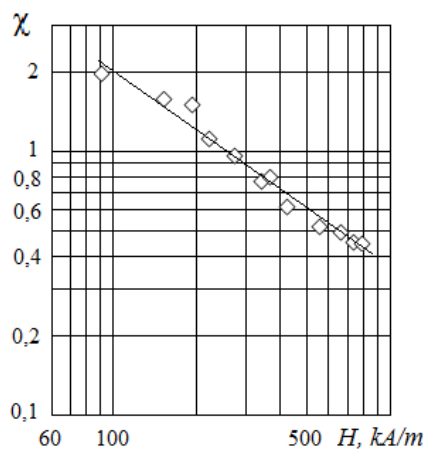

Fig.6. Dependence of the magnetic susceptibility of magnetite particles on magnetic field intensity

\section{Conclusion}

In this paper corresponding optical-mechanical system of positioning of the measuring sensor (Hall) and the studied sample is recommended. On the received concentration dependences of magnetic susceptibility of powder samples existence of limited line sections is confirmed that gives the chance to define susceptibility of particles. 


\section{Acknowledgement}

The research is conducted with financial support from RFBR within the frame works of research project No 16-38-60034 mol_a_dk and from Russian Federation Ministry of Education and Science No 9.9626.2017.

\section{References}

1. S. Das, S. Chakraborty, S.K. Mitra. Microfluidics and Nanofluidics, 13(5), 799-807 (2012)

2. M. Kawano, H. Watarai, Analytical and Bioanalytical Chemistry. 403(9), 2645-2653 (2012)

3. K. Nandy, S. Chaudhuri, R. Ganguly, I.K. Puri. JMMM. 320, 1398-1405 (2008)

4. V. Murariu, J. Svoboda. Phys. Separ. in Sci. and Engineering. 12(1), 1-11 (2003)

5. A.A. Sandulyak, A.V. Sandulyak, B.M. Belgacem Fethi, D.O. Kiselev. Journal of Magnetism and Magnetic Materials. 401, 902-905 (2016)

6. Patent USA №4492921. Sandulyak A.V., Garaschenko V.I., Korkhov O.J. Method of Determining the Quantity of Solid Fraction of Ferromagnetic Matter in a Fluid. 1985.

7. J.C.P. Klaasse. The Faraday balance, Van der Waals Zeeman Institute, November 1999, URL: https://iop.fnwi.uva.nl/cmp//klaasse/fdb.html.

8. V.I. Chechernikov. Magnitnye izmereniya (Magnetic Measurements), Moscow: Izd. MGU, 1969, $388 \mathrm{p}$.

9. A.Y. Dorfman, S.K. Sidorov. Zh. Eksp. Teor. Fiz. 9(1), 25 (1939)

10. V.R. Karasik. Fizika i tekhnika sil'nykh magnitnykh polei (Physics and Technology of Strong Magnetic Fields), Moscow: Nauka, 1964, 348 p.

11. R.D. Heyding, J.B. Taylor, M.L. Hair. Rev. Sci. Instrum. 32(2), 161-163 (1961)

12. D. Govindarajan, R. Gopalakrishnan. International J. Minerals, Metallurgy and Materials. 16(3), 349-354 (2009)

13. R. Gopalakrishnan, S. Barathan, D. Govindarajan, American J. Materials Science. 2(1), 32-36 (2012)

14. A.V. Sandulyak. Magnetic and filtration purification of liquids and gases. Moscow: Chemistry, $\quad 1988, \quad 136 \quad$ p. https://dlib.rsl.ru/viewer/01001440011\#?page=136

15. A.V. Sandulyak, A.A. Sandulyak, V.A. Ershova. Technical Physics. 54(5), $743-$ 745 (2009)

16. A.A. Sandulyak, V.A. Ershova, D.V. Ershov, A.V. Sandulyak. Solid State Physics. 52(10), 1967-1974 (2010)

17. A.V. Sandulyak, A.A. Sandulyak, V.A. Ershova. Doklady Physics. 52(4). 179$181(2007)$

18. A.V. Sandulyak, A.A. Sandulyak, M.N. Polismakova, D.O. Kiselev, D.A. Sandulyak, V.A. Ershova. MATEC Web of Conferences 10, 01007 (2017)

19. A.V. Sandulyak, A.A. Sandulyak, M.N. Polismakova, V.A. Ershova, D.A. Sandulyak, D.O. Kiselev. Applied Physics, System Science and Computers, Lecture Notes in Electrical Engineering 428, 77-83 (2016)

20. A.A. Sandulyak, A.V. Sandulyak, M.N. Polismakova, D.O. Kiselev, V.A. Ershova , D.A. Sandulyak. Instrum. and Experim. Tech. 61(1), 123-126 (2018)

21. A.A. Sandulyak, D.A. Sandulyak, M.N. Polismakova, A.V. Sandulyak, D.O. Kiselev, V.A. Ershova. J. Eng. Phys. and Thermophys. 90(4), 845-850 (2017)

22. D.A. Sandulyak, A.A. Sandulyak, D.O. Kiselev, A.V. Sandulyak, M.N. Polismakova, M.A. Kononov, V.A. Ershova. Meas. Tech. 60(9), 928-933 (2017) 